\title{
7 Zustellroboter als Lösung für die letzte Meile in der Stadt?
}

Bert Leerkamp, Aggelos Soteropoulos, Martin Berger

1. Einleitung

108

2. E-Commerce und Lieferverkehre nehmen zu

108

3. Neue Zustellkonzepte für die letzte Meile

4. Betriebskonzepte automatisierter Fahrzeuge in der Logistik

5. Zustellroboter

5.1 Zustellroboter für den Betrieb auf Gehwegen

5.2 Zustellroboter für den Betrieb auf öffentlichen Straßen

6. Ausgewählte Beispiele von Tests mit Zustellrobotern

7. „Gehwegtaugliche“ Zustellroboter als Lösung für die letzte Meile?

8. Planerische Implikationen

9. Fazit

Ein Interview von Martin Berger und Aggelos Soteropoulos mit Bert Leerkamp

Bert Leerkamp

Bergische Universität Wuppertal, Lehr- und Forschungsgebiet für Güterverkehrsplanung und Transportlogistik

leerkamp@uni-wuppertal.de

Aggelos Soteropoulos

TU Wien, future.lab Research Center und Forschungsbereich Verkehrssystemplanung (IVS)

aggelos.soteropoulos@tuwien.ac.at

Martin Berger

TU Wien, Forschungsbereich Verkehrssystemplanung (IVS)

martin.kp.berger@tuwien.ac.at

(C) Der/die Autor(en) 2021

M. Mitteregger et al. (Hrsg.), AVENUE21. Politische und planerische Aspekte der automatisierten Mobilität, https://doi.org/10.1007/978-3-662-63354-0_7 


\section{EINLEITUNG}

Der städtische Güterverkehr nimmt eine immer wichtigere Rolle im Bereich der urbanen Mobilität sowie auch in der städtischen Verkehrspolitik ein, da der E-Commerce boomt: Die Digitalisierung bringt es mit sich, dass immer öfter Waren über das Internet bestellt werden, wodurch sich auch der Lieferverkehr stark erhöht hat (vgl. Muschkiet/Schückhaus 2019: 358; Deutsche Bundesregierung 2019: 44f.). Es bedarf daher neuer nachhaltiger Lösungen und Konzepte in der urbanen Logistik, wobei besonders die letzte Meile infolge fehlender Bündelung und groBer Aufwände eine wesentliche Herausforderung darstellt (vgl. Gerdes/Heinemann 2019: 399; Buthe et al. 2018: 30; Lierow/Wisotzky 2019). Gleichzeitig entstehen neue Zustellformen wie etwa Zustellroboter (vgl. Baum et al. 2019: 2455; Jennings/Figliozzi 2019: 317), denen ein groBes Potential für die letzte Meile zugeschrieben wird.

Bislang prägen die Entwicklung und Anwendung von Zustellrobotern vor allem Logistikunternehmen und Technologieentwickler, ohne dass die kommunale Perspektive in den Blick genommen wird. So stellt sich die Frage, ob und wie weit Zustellroboter in urbanen öffentlichen Räumen überhaupt verträglich sind. Dies ist umso wichtiger, da der öffentliche Raum bereits jetzt durch neue Mobilitätsformen, Klimawandelanpassung, steigende Bevölkerungszahlen in Städten und einen damit einhergehenden Nutzungsdruck zunehmend stärker beansprucht wird. Gerade wenn die automatisierten Zustellroboter auf Fußwegen und in Fußgängerzonen unterwegs sind, sind Nutzungs- und Interessenskonflikte vorprogrammiert (vgl. Buthe et al. 2018: 121). Vor dem Hintergrund der notwendigen Transformation des öffentlichen Raums vom Transit- zum Aufenthaltsraum werden daher die vielfältigen Implikationen von Zustellrobotern diskutiert und (verkehrs)politische und planerische Handlungsspielräume aufgezeigt.

\section{E-COMMERCE UND LIEFERVERKEHRE NEHMEN ZU}

Der Onlinehandel - kurz „E-Commerce“ bzw. „Distanzhandel“ - boomt. Aber auch Lieferangebote des stationären Handels führten in den letzten Jahren zu einem stetigen Marktwachstum für Kurier-, Express- und Paket (KEP)-Dienste. In Österreich beispielsweise nahm die Anzahl

Abbildung 1: Entwicklung des österreichischen Paketmarkts in Millionen Stück 2009 bis 2018

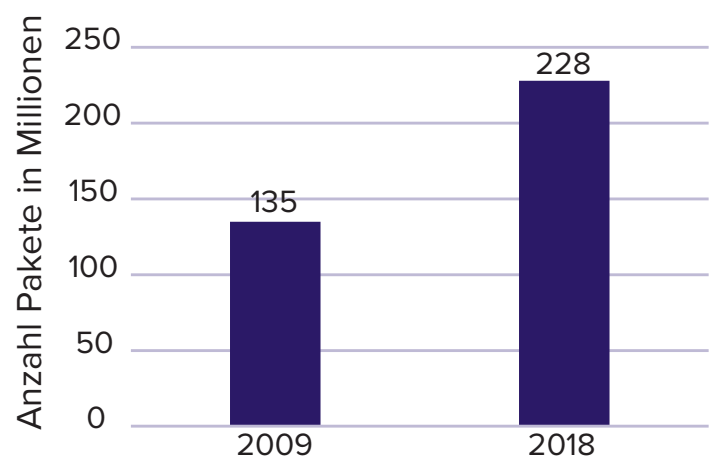

Quelle: Umundum (2020: 151) 
Abbildung 2: Umsatzentwicklung im E-Commerce in Österreich 2015 bis 2019

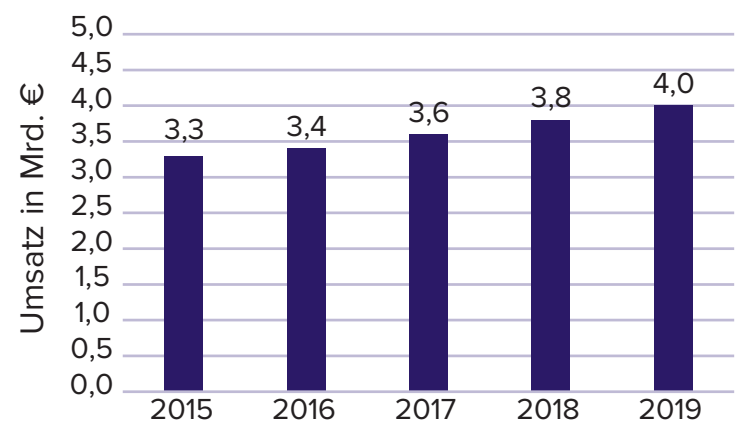

Quelle: WIFO (2019: 15)

der Pakete zwischen 2009 und 2018 um etwa 69 Prozent zu, weltweit hat sich die Anzahl der Pakete allein zwischen 2014 und 2018 mehr als verdoppelt. Ähnliche Entwicklungen sind auch in Deutschland erkennbar (vgl. BIEK 2020: 11). Prognosen gehen sowohl in Österreich als auch weltweit von einer weiter steigenden Anzahl an Paketen aus (vgl. Umundum 2020: 151; Buchholz 2019). Auch der Umsatz des E-Commerce in Österreich verzeichnete in den letzten Jahren, zwischen 2015 und 2019, einen deutlichen Anstieg von 21 Prozent, wodurch der Onlineanteil am gesamten Einzelhandelsumsatz in Österreich derzeit schon bei über 5 Prozent liegt (Abb. 1; vgl. WIFO 2019: 15).

Als Folge steigender Lieferverkehre erhöht sich auch der Handlungsbedarf von Städten und Gemeinden, proaktiv Strategien und Konzepte für den kommunalen Güterverkehr zu entwickeln, aber auch Maßnahmen zu planen und umzusetzen (vgl. Schönberg et al. 2018: 4): Da im städtischen Gütertransport sehr viele Endnutzerlnnen beliefert werden, ergibt sich eine hohe Anzahl an kleinen Einzellieferungen, die wiederum zu vielen gefahrenen Kilometern führt (vgl. Wirtschaftsagentur Wien 2016: 5). Daraus resultieren eine steigende Feinstaubbelastung, $\mathrm{CO}_{2}$ - und Lärmemissionen (vgl. Muschkiet/Schückhaus 2019: 366), aber vor allem auch Konflikte im öffentlichen Raum, die sich vielschichtig in Flächenkonkurrenzen, Gefährdungen von Menschen, aber auch in der „Kommerzialisierung des öffentlichen Raums“ manifestieren.

Aufgrund der prognostizierten Steigerung des Sendungsvolumens - in Österreich beispielsweise wird für das Jahr 2020 eine Zunahme der Anzahl der Pakete von etwa 14 Prozent im Vergleich zum Jahr 2018 erwartet - wird die Bedeutung der kostenintensiven letzten Meile mit allen negativen Effekten auf den öffentlichen Raum weiter steigen (vgl. Leerkamp 2017: 12; Umundum 2020: 151).

\section{NEUE ZUSTELLKONZEPTE FÜR DIE LETZTE MEILE}

Die letzte Meile, d. h. die Zustellung von Waren beim Kunden vor Ort, stellt noch immer eines der zentralen Probleme im städtischen Güterverkehr dar: Der Auslastungsgrad der Transportträger bei der Ver- und Entsorgung sinkt mit der Nähe zum Ziel und die Bündelung wird im letzten Abschnitt der Lieferkette immer schwieriger (vgl. Just 2018: 5; open4innovation 2019). Mehr als 50 Prozent der Kosten bei der Paketlieferung fallen auf der letzten Meile an (vgl. Schnedlitz et al. 2013: 251; Schocke 2019). Insbesondere außerhalb des effektiven Lieferzeitfensters, d. h. dann, 
wenn die Wahrscheinlichkeit sinkt, dass Personen zu Hause sind, besteht zusätzlich eine geringe Effizienz durch erforderliche Mehrfachzustellungen.

Neue Logistikkonzepte werden im Spannungsfeld zwischen betriebswirtschaftlicher Effizienz einerseits sowie kommunalen Ansprüchen der Verkehrsvermeidung und -verlagerung und umfeldverträglicher Zustellung andererseits umgesetzt: Die Konzepte sollen helfen, „Bündelungseffekte" zu erzielen, den Stopp-Faktor im Endkundengeschäft zu erhöhen und den Transportaufwand zu reduzieren (Buthe et al. 2018: 30). Ein vielversprechendes Logistikkonzept ist die Belieferung von Sammelpunkten - sogenannten City-Hubs - in der städtischen Kernzone durch wenige, große Lkws aus der Peripherie. Von dort gelangen die Pakete auf der letzten Meile entweder direkt zu den Kundlnnen oder zu Mikrodepots und Paketboxen. Für die letzte Meile eignen sich verschiedene Fahrzeug- und Antriebskonzepte oder die Zustellung mit (E-)Lastenrädern (vgl. Wittenbrink et al. 2016: 79f.; Leerkamp 2019: 21; Gerdes/Heinemann 2019: 406).

Automatisierung und Digitalisierung und darauf aufbauende Zustellkonzepte bewirken einen Innovationsschub (vgl. Umundum 2020: 157). Neben Zustelldrohnen erfolgen in jüngster Zeit Testanwendungen zu elektrischen Zustellrobotern, sogenannte automatisierte „Delivery Bots“, beispielsweise in den USA, aber auch in Europa. Die Last-Mile-Zustellung wird häufig als eine der ersten Anwendungsbereiche des automatisierten Fahrens betrachtet, da diese Zustellroboter mit niedrigen Geschwindigkeiten und in einer möglicherweise einfachen Einsatzumgebung (ODD - Operational Design Domain), beispielsweise auf einem Gehweg in einem Wohngebiet am Stadtrand, unterwegs sind (vgl. Soteropoulos et al. 2020; Mitteregger et al. 2020; Leitner et al. 2018: 22).

\section{BETRIEBSKONZEPTE AUTOMATISIERTER FAHRZEUGE IN DER LOGISTIK}

Automatisierte Fahrzeuge im Bereich der Logistik zu verwenden, ist keineswegs neu, sondern erfolgt bereits schon seit Längerem vor allem in der innerbetrieblichen Logistik. Bereits seit den 1950er Jahren sind automatisierte Fahrzeuge für den Transport von Gütern in der Produktion oder in logistischen Systemen im Einsatz, dies vor allem für den Transport ohne Fahrerln (1) im Innenbereich bzw. innerhalb abgegrenzter Gebäude, (2) im privaten Außenbereich, z. B. auf Betriebsgeländen oder Containerterminals, oder (3) in gefährlichen oder kaum zugänglichen Gebieten (vgl. Flämig 2015: 378; Hörl et al. 2019: 35; Paddeu et al. 2019: 9ff.; Hofer et al. 2018: 11ff.).

Innerwerkliche Verkehre sind auch heute noch der typische Anwendungszweck automatisierten Fahrens in der Logistik, z. B. bei der autonomen Hoflogistik der Österreichischen Post, und unterliegen dabei spezifischen Rahmenbedingungen bei Infrastruktur und Prozessen (vgl. Clausen 2017: 16; Muschkiet/Schückhaus 2019: 374; Umundum 2020: 156). Infolge von Fortschritten im Bereich der Automatisierung und Digitalisierung gerät nun auch die Distributionslogistik zunehmend in den Anwendungsblick. Hierbei wird neben automatisierten Lkws im Fernverkehr (z. B. Platooning - obwohl diesbezügliche Tests zum Teil auch, z. B. durch Daimler, gestoppt wurden; vgl. Daimler 2019) und automatisierten Zustellkonzepten, bei denen die Zustellung per Drohnen erfolgt, derzeit auch die Zustellung mit Zustellrobotern in öffentlichen Räumen von Städten und Gemeinden erprobt (vgl. Baum et al. 2019: 2457; Howell et al. 2020: 36; Schröder et al. 2018: 7; Hofer et al. 2018: 14ff.). Abbildung 3 gibt einen Überblick der Betriebskonzepte automatisierter Fahrzeuge im Bereich der Logistik. 
Abbildung 3: Überblick der Betriebskonzepte automatisierter Fahrzeuge in der Logistik

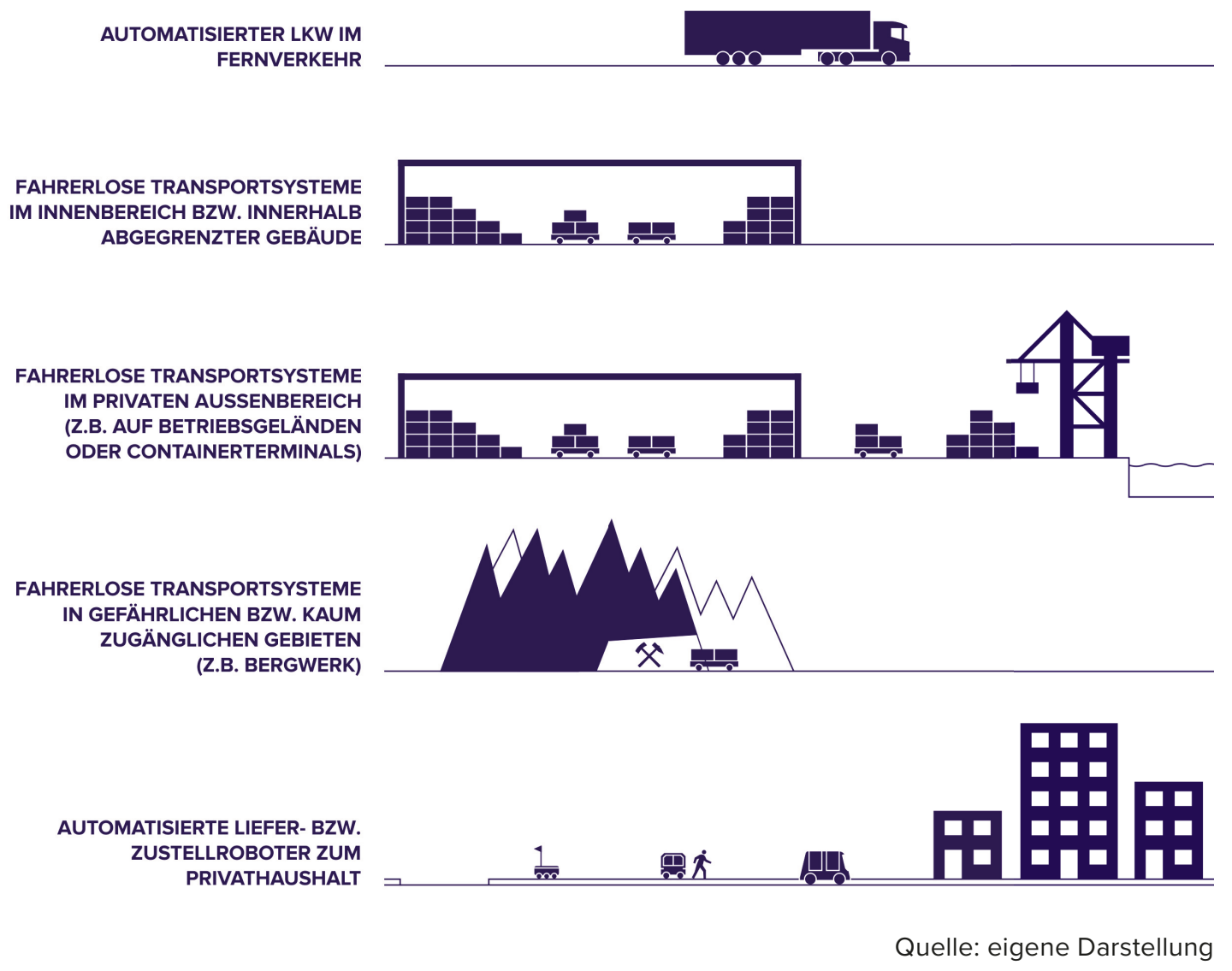

\section{ZUSTELLROBOTER}

Bei einem Zustellroboter handelt es sich um ein fahrerloses, häufig elektrisches Transportmittel, das die letzte Meile von einem innerstädtischen Lagerhaus oder einem stationären Händler zu KundInnen in einem definierten, freigegebenen Bereich übernimmt (vgl. Vogler et al. 2018: 152; Leerkamp 2017: 17). Einsatzzwecke sind beispielsweise Spezialsendungen, die flexibel, schnell und günstig in einer lokalen Umgebung ausgeliefert werden müssen, „Same-Day Delivery“ oder „Same-Hour Delivery“, Essenslieferungen oder Hauslieferungen von medizinischen Produkten (vgl. Hofer et al. 2018: 17). Zudem bestehen auch Logistikkonzepte, die vorsehen, dass Lieferwagen die Zustellroboter in ein größeres Zustellgebiet bringen, die von dort aus die Endzustellung an die Kundlnnen übernehmen (vgl. Jennings/Figliozzi 2019: 321; DHL 2014: 32).

Zustellroboter werden derzeit von zahlreichen Unternehmen entwickelt, wobei grundsätzlich unterschiedliche Arten bestehen (vgl. Baum et al. 2019: 2457; Steer 2020: XVIII sowie folgende Abb. 4). 
Abbildung 4: Überblick zu unterschiedlichen Zustellrobotern

\section{Automatisierte Liefer- bzw. Zustellrobo- ter für den Betrieb auf Gehwegen}

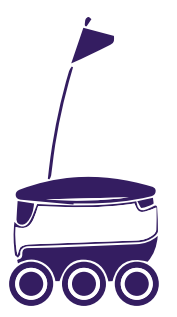

ohne „Follow-Me“Funktion

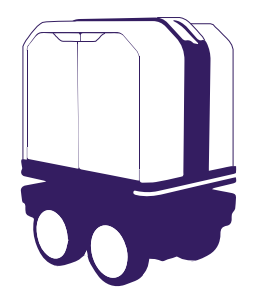

mit „Follow-Me“Funktion
Automatisierte Liefer- bzw. Zustellroboter für den Betrieb auf öffentlichen Straßen

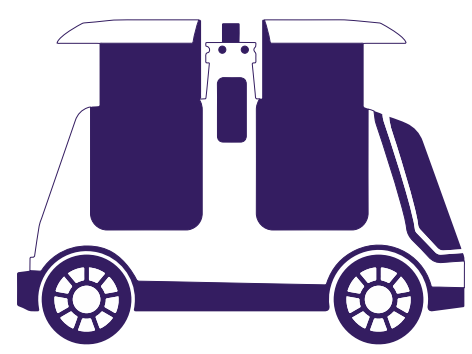

Quelle: eigene Darstellung

\subsection{ZUSTELLROBOTER FÜR DEN BETRIEB AUF GEHWEGEN}

Dieser Typ von Zustellroboter eignet sich nicht, um auf Fahrbahnen öffentlicher Straßen zu verkehren, sondern ist auf Gehwegen und in Fußgängerzonen unterwegs. Dort ist die Geschwindigkeit auf max. $6 \mathrm{~km} / \mathrm{h}$ limitiert. Aktuelle Einsatzbereiche dieses Zustellroboters sind Büroparks (z. B. in Mountain View, USA) oder andere Sonderareale - denn dort liegen die Anforderungen für das automatisierte Fahrsystem weit nicht so hoch wie im öffentlichen Straßenraum (vgl. Hern 2018). Zudem verfügen sie in der Regel meist über ein geringes Ladevolumen (vgl. Leerkamp 2017: 17).

\section{a) Zustellroboter für die autonome Zustellung}

Diese Zustellroboter sind dabei für die autonome Lieferung von einzelnen Sendungen in kurzen Zeitfenstern (20-30 Minuten) gerade innerhalb von dichten Stadtquartieren geeignet. Zu den Herstellern dieser Zustellroboter zählen u. a. Starship Technologies, Kiwi und Marble. In der Regel besitzen die Roboter einen kleinen Behälter, in welchem eine Sendung eingelegt werden kann (vgl. Marks 2019: 13ff.). Der Zustellroboter von Starship Technologies beispielsweise kann in der gegenwärtigen Auslegung maximal zwei Pakete à $35 \times 34 \times 15 \mathrm{~cm}$ transportieren (vgl. Ninnemann et al. 2017: 86). Ist der Zustellroboter beim Kunden bzw. der Kundin angekommen, kann dieser den Deckel des Behälters vor seiner Haustür mit einer Einmal-PIN öffnen, die ihm/ihr per Smartphone zugeschickt wird (vgl. Vogler et al. 2018: 152).

\section{b) Zustellroboter mit „Follow-me“-Funktion}

Außerdem können Zustellroboter, die auf Gehwegen fahren, auch mittels Follow-me-Funktion die Distributionslogistik unterstützen. Hierbei folgt der Zustellroboter mit der Sendung dem/ der Zustellerln bzw. Kommissioniererln, der bzw. die dadurch entlastet wird. Ein Beispiel ist der Zustellroboter PostBOT der Firma Effidence S.A.S, der gemeinsam mit der Deutschen Post entwickelt wurde. Im Gegensatz zu den Zustellrobotern, die ihre Zustellung autonom ausführen, sind Follow-me-Modelle meist etwas größer. So besitzt der PostBOT beispielsweise sechs Sendungsbehälter und ermöglicht den Transport einer Sendungsmenge von bis zu $150 \mathrm{~kg}$ (vgl. Gerdes/Heinemann 2019: 411). 


\subsection{ZUSTELLROBOTER FÜR DEN BETRIEB AUF ÖFFENTLICHEN STRASSEN}

Zustellroboter, die auf öffentlichen Straßen fahren, sind mit einer Geschwindigkeit von max. 40 bis $50 \mathrm{~km} / \mathrm{h}$ unterwegs und verfügen aufgrund ihrer Größe über ein größeres Ladevolumen als die zuvor genannten Modelle. Der typische Einsatzbereich ist die autonome Zustellung einzelner Sendungen in kurzen Zeitfenstern (20-30 Minuten) gerade innerhalb von Gebieten hoher Kundendichte. Beispiele für Zustellroboter dieses Typs sind der Nuro R1 und sein Nachfolger Nuro R2, Robomart oder Udelv (Baum et al. 2019: 2458; Marks 2019: 22). Erst kürzlich erhielt der Nuro $R 2$ vom Verkehrsministerium in den USA die erste US-weite temporäre Ausnahmegenehmigung („Temporary Exemption“), um das Fahrzeug auf öffentlichen Straßen ohne Fahrerln zu testen (USDOT 2020: 5). Diese Fahrzeuge eignen sich nicht nur für die Zustellung der letzten Meile: Waymo gab beispielsweise vor Kurzem bekannt, dass im Rahmen ihres laufenden Testbetriebs in Chandler, Arizona, ihre Fahrzeuge ebenso für die Lieferung von Paketen von lokalen UPS-Geschäften zu einer UPS-Paketsortieranlage eingesetzt werden - eine Paketzustellung an Kundlnnen ist jedoch noch nicht angedacht (UPS 2020). Dabei ist in den USA in den typischen suburbanen Bereichen mit sehr breiten Straßen, Einfamilienhäusern und wenig Fußgänger- und Radfahrerlnnen, in denen die Fahrzeuge unterwegs sind (z. B. Nuro R2 in Scottsdale, Arizona, oder Houston, Texas; vgl. Nuro 2020), die Abholung der Ware aus dem Fahrzeug durch die Kundlnnen an der Bordsteinkante bzw. auf speziellen Flächen, an denen der Fahrroboter halten darf (sogenannte "self-driving pick-ups“), wesentlich leichter möglich als im europäischen, oftmals durch engere Straßen und höhere Dichte gekennzeichneten Pendant.

\section{AUSGEWÄHLTE BEISPIELE VON TESTS MIT ZUSTELLROBOTERN}

Zahlreiche Länder testen Zustellroboter in Pilotversuchen. Während in Deutschland, Österreich und der Schweiz mehrheitlich Zustellroboter ausprobiert wurden, die nicht für den Verkehr auf öffentlichen Straßen gedacht sind, sondern allein auf Gehwegen und in Fußgängerzonen eingesetzt werden (vgl. Baum et al. 2019: 2459; Hofer et al. 2018: 17), gab es in den USA hingegen bereits auch Tests und Pilotversuche mit Zustellrobotern, die auf öffentlichen Straßen unterwegs sind. Tabelle 1 gibt einen Überblick ausgewählter Beispiele.

Mit Ausnahme der Schweiz lag das Testgebiet meistens in der Innenstadt bzw. Geschäftsquartieren größerer Städte. Der Test des Follow-me-Zustellroboters fand in der Kleinstadt Bad Hersfeld, jedoch auch eher im Zentrum bzw. Geschäftsquartier statt. In den USA waren die Testfelder des fahrbahntauglichen Modells öffentliche Straßen in Wohn- bzw. Gewerbegebieten am Stadtrand bzw. in suburbanen Bereichen.

Die Tests dienten dazu, zu überprüfen, wie sicher, praktikabel und wirtschaftlich die Zustellroboter in der Praxis aus der Perspektive der Logistikunternehmen eingesetzt werden können. Testanwender sind neben der Post auch KEP-Dienste sowie Einzelhandels-, Restaurant- und Supermarktketten. Es lässt sich feststellen, dass keine umfassende Evaluierung und wissenschaftliche Begleitung abseits von unternehmensspezifischen Erkenntnisinteressen erfolgt sind. Wichtige Fragestellungen wie Ziel- und Nutzungskonflikte im öffentlichen Straßenraum und insbesondere die Interaktion mit anderen Verkehrsteilnehmerlnnen waren nicht Gegenstand der Tests. Ausnahme bilden die Tests in der Schweiz, wo diese Aspekte zwar etwas detaillierter betrachtet, aber nicht umfassend wissenschaftlich evaluiert wurden. 


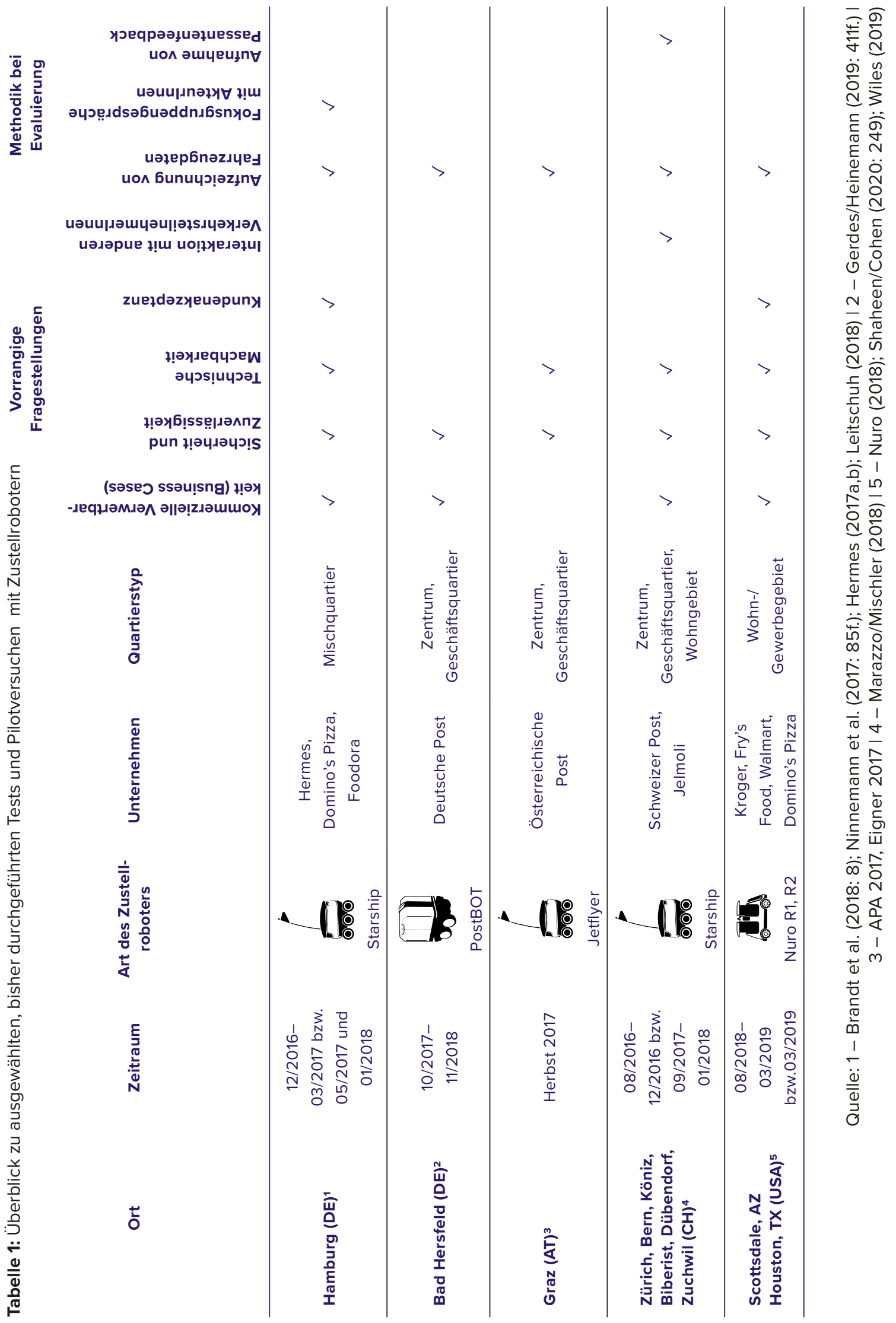




\section{7. „GEHWEGTAUGLICHE“ ZUSTELLROBOTER ALS LÖSUNG FÜR DIE LETZTE MEILE?}

Wie die Analyse zeigt, konzentriert sich die Entwicklung weltweit mehrheitlich auf Roboter, die auf Gehwegen und/oder in Fußgängerzonen unterwegs sind (vgl. Baum et al. 2019: 2459), sodass sich die folgenden Ausführungen auf diesen Anwendungsfall fokussieren (siehe auch Abb. 5).

Wie bereits ausgeführt, werden große Hoffnungen in „gehwegtaugliche“ Zustellroboter in Stadtquartieren hoher Dichte für die Zustellung einzelner Sendungen in kurzen Zeitfenstern gesetzt. Ein größeres wirtschaftliches Potential lässt sich erst dann ausschöpfen, wenn die Roboter nicht mehr mit menschlichen BegleiterInnen unterwegs sein müssen, sondern aufgrund rechtlicher Erfordernisse allenfalls von einer/m menschlichen Operatorln aus der Ferne überwacht werden müssen (vgl. Hermes 2017b), wobei noch ungeklärt ist, welche Anforderungen der Gesetzgeber an diese Überwachung stellen wird und welches Spannungsfeld sich bezüglich der Genauigkeit und Art der Überwachung und des daraus resultierenden Personalaufwands $-z$. B. (keine) gleichzeitige Überwachung von mehreren Fahrzeugen - ergeben wird. Zustellroboter mit Follow-me-Funktion könnten hingegen die körperliche Belastung der Zustellpersonen reduzieren und besitzen zudem den Vorteil, dass die Zustellperson bei Störungen bzw. Konflikten eingreifen könnte. Haben die Fahrzeuge einen elektrischen Antrieb, so könnte dies mit einer Reduktion der $\mathrm{CO}_{2}$ - und Lärmemission verbunden sein, ohne dass bislang fundierte Wirkungsanalysen vorhanden sind. Lediglich Jennings und Figliozzi stellen in vergleichenden Simulationen fest, dass die Kombination von Zustellrobotern und Lieferfahrzeugen gegenüber der Belieferung ausschließlich mit dem Lieferwagen die Lieferzeiten senkt sowie gefahrene Kilometer und Kosten insbesondere bei hoher Kundendichte reduziert (vgl. Jennings/Figliozzi 2019: 324). Aus der Logik der Zustellerunternehmen ergibt sich aus der Gegenüberstellung der Kosten für Anschaffung und Betrieb eines Zustellroboters im Verhältnis zum generierbaren Transporterlös für die letzte Meile ein betriebswirtschaftlicher Erfolg (vgl. Hofer et al. 2018: 48). Zum gegenwärtigen Zeitpunkt sind Zustellroboter noch zu teuer, weshalb es im deutschsprachigen Raum bislang bei Pilotversuchen geblieben ist (vgl. Hermes 2017b, Marazzo/Mischler 2018: 1; Wittenhorst 2019). Auch langfristig bleibt offen, inwieweit ein positives Kosten-Nutzen-Verhältnis in Abhängigkeit beispielsweise von der Kundendichte, Siedlungsdichte, dem Anwendungszweck etc. gegeben ist, da die Ladungsmenge der Zustellroboter relativ gering ist (vgl. Hofer et al. 2018: 48). Hinzu kommen auch noch Sicherheitsaspekte (hinsichtlich des Diebstahls von Fahrzeugen) oder Vandalismus, die bei einem Einsatz der Fahrzeuge berücksichtigt werden müssten (vgl. Paddeu et al. 2019: 32; Kunze 2016: 292; Hofer et al. 2018: 18).

Außerdem zeigen die Pilotversuche konkrete technische Probleme wie eine limitierte Akkuleistung und Anwenderfreundlichkeit der Benutzeroberfläche („user interface“) etc. auf. Zusätzlich erfordern die Zustellroboter ein leistungsfähiges LTE-Mobilfunknetz, welches nicht immer flächendeckend zur Verfügung steht (Hermes 2017a; Marrazzo/Mischler 2018: 2). Diese Probleme dürften in naher Zukunft aber lösbar sein.

Anders sieht es bei den Herausforderungen aus, welche die Interaktion von Mensch und Zustellroboter im öffentlichen Raum betreffen, wo es um mangelnde Akzeptanz, konkrete Störwirkungen und Gefährdungen geht:

- Zustellroboter schränken die Bewegungsfreiheit aller FußgängerInnen, insbesondere aber mobilitätseingeschränkter Personen - vor allem mit Gehhilfen (vgl. Lenthang 2019; Hofer et al. 2018: 48) -, Kinder und Älterer ein (vgl. Marks 2019: 14); 
- $\quad$ sie können im öffentlichen Raum eine Gefährdung darstellen, falls die FußgängerInnen aufgrund mangelnder Reaktion, Beweglichkeit etc. nicht rechtzeitig ausweichen können, um eine Kollision zu verhindern, oder unerwartet ihre Gehrichtung wechseln.

- Dieser Effekt verstärkt sich zudem auf schmalen Gehsteigen oder Fußgängerübergängen mit einer hohen Anzahl an FußgängerInnen, unterschiedlichen Gehgeschwindigkeiten und allseitig gerichteten Bewegungsmustern der PassantInnen (vgl. Leerkamp 2017:17; Marazzo/ Mischler 2018: 4; Hsu 2019).

Besonders in folgenden Fahrsituation, die Zustellroboter permanent zu bewältigen haben, sind Konflikte vorprogrammiert (vgl. Keesmaat 2020: 9; Groot 2019: 64):

- Umfahren von Hindernissen,

- Queren einer Fahrbahn an einem Fußgängerüberweg oder einer Lichtsignalanlage, wobei beispielsweise Marazzo und Mischler (2018: 4) berichten, dass die Grünzeit für das Queren des Zustellroboters und der Begleitperson nicht ausreichend war,

- Überholen von langsam gehenden FußgängerInnen,

- Kontakt mit einem spielenden Kind oder einer Gruppe von Kindern oder größeren Pulks von Personen sowie

- Fahren auf/von einem Bordstein oder einer Rampe (vgl. Leerkamp 2017: 17).

Automatisiertes Fahren des Zustellroboters im dichten Mischverkehr, das eine Interaktion mit zahlreichen und unterschiedlichen Verkehrsteilnehmerlnnen bedingt, ist eine relativ komplexe Fahraufgabe und erlaubt nur geringe Geschwindigkeiten (vgl. Hofer et al. 2018: 49; vgl. Abb. 6). Weitgehend ungeklärt ist auch, welche technischen Anforderungen aufgrund der oben genannten Konflikte bei Fahrsituationen an die Beschaffenheit der Gehwege, insbesondere hinsichtlich Bordabsenkungen, Furten an Knotenpunkten oder der farblichen Unterscheidung von Geh- und Radwegen gestellt werden.

Aber auch beim Auslieferungsprozess existieren mehrere Barrieren: So ist etwa im Logistikkonzept zu lösen, wie Lieferungen an Kundlnnen gelangen, die nicht zu Hause sind Auch wie aufgrund der fehlenden Fähigkeit des Zustellroboters, Treppen zu überwinden - die Zustellung in mehrgeschossigen Gebäuden erfolgt, muss geklärt werden. Hier fehlen bislang „Drop-off“Boxen bzw. Paketboxen, die ebenerdig für die Lieferroboter erreichbar, gegenwärtig jedoch nur vereinzelt vorhanden sind.

Derzeit sind die rechtlichen Rahmenbedingungen, um Zustellroboter in öffentlichen Straßen einzusetzen, in Deutschland und der Schweiz im Vergleich zu manchen Bundesstaaten in den USA (vgl. Jennings/Figliozzi 2019) relativ rigide und beschränkend. Ohne Begleitperson kann kein automatisierter Zustellroboter im öffentlichen Raum betrieben werden. In Deutschland wird hierfür gemäß Straßenverkehrsordnung (StVO) und Straßenverkehrszulassungsordnung (StVZO) meist eine Ausnahmegenehmigung erteilt, die konkrete Bedingungen und Auflagen für den Betrieb von Zustellrobotern enthält (vgl. Brandt et al. 2018: 7). Auch das Thema Datenschutz spielt eine Rolle, da Zustellroboter mit bildgestützter Sensorik die Umgebung erfassen und dabei, um Objekte zu erkennen, auch „kritische“ personenbezogene Daten anderer VerkehrsteilnehmerInnen erheben. Hier geht es um die Vereinbarkeit mit den Inhalten nationaler Datenschutzgesetze sowie der europäischen Datenschutzgrundverordnung (DSGVO), vor allem Artikel 25 (Datenschutz durch Technikgestaltung). Dabei muss gewährleistet sein, dass im Rahmen der Objekterkennung anderer VerkehrsteilnehmerInnen durch die Zustellroboter 
grundsätzlich nur jene personenbezogenen Daten verarbeitet werden, die für den Zweck erforderlich sind (vgl. Brandt et al. 2018: 8; Hoffmann/Prause 2018: 11). Letzteres gilt dabei auch hinsichtlich einer möglichen videogestützten Überwachung des Fahrtenverlaufs der Zustellroboter durch die Operatorlnnen aus der Ferne. Insbesondere die Auflage einer ständigen Begleitung des Zustellroboters im öffentlichen Raum durch eine Begleitperson macht für die Unternehmen eine Nutzung von solchen Zustellrobotern unrentabel. Vielmehr dienen die Tests mit automatisierten Zustellrobotern vor allem eher der Sammlung von praktischen Erfahrungen mit neuen Technologien als Basis für das Ausloten der Handlungsspielräume aus Sicht der Unternehmen (vgl. Ninnemann et al. 2017: 138), um sich in weiterer Folge gezielter für die Interessen einsetzen zu können und beispielsweise eine Zulassung ohne Begleitperson zu erreichen. Dieses Ansinnen steht aber im Gegensatz zu den großen Herausforderungen im öffentlichen Raum.

Abbildung 5: Überblick der Stärken, Schwächen, Chancen und Risiken von auf dem Gehweg fahrenden automatisierten Liefer- bzw. Zustellrobotern für die letzte Meile

\begin{tabular}{|c|c|}
\hline Stärken & Schwächen \\
\hline $\begin{array}{l}\text { - Optimierung der Zustellung einzelner Sendungen } \\
\text { in kurzen Zeitfenstern } \\
\text { - Effizienzsteigerung durch Unterstützung des Zu- } \\
\text { stellers (parallele Ausführung anderer Aufgaben) } \\
\text { - Reduzierung von } \mathrm{CO}_{2} \text { - und Lärmemissionen } \\
\text { durch elektrischen Antrieb }\end{array}$ & $\begin{array}{l}\text { - technische Probleme: Akkuleistung, „user inter- } \\
\text { face“, Flexibilität des Systems, komplexer Misch- } \\
\text { verkehr } \\
\text { - mangelnde Wirtschaftlichkeit durch Erfordernis } \\
\text { einer begleitenden Person, hohen Anschaffungs- } \\
\text { preis, geringe Zuladung } \\
\text { - fehlenden Fähigkeit, Treppen zu überwinden } \\
\text { - } \text { spezielle Drop-off-Boxen bei dem/r Empfängerln } \\
\text { nötig, wenn niemand zu Hause ist }\end{array}$ \\
\hline Chancen & Risiken \\
\hline $\begin{array}{l}\text { - Überwachung durch menschliche/n Operatorln } \\
\text { aus der Ferne und damit Ausschöpfung von wirt- } \\
\text { schaftlichem Potential möglich } \\
\text { - Reduzierung körperlicher Belastung von Zustell- } \\
\text { personen durch Zustellroboter mit Follow-me- } \\
\text { Funktion } \\
\text { - in Kombination mit Lieferfahrzeugen Reduktion } \\
\text { von Lieferzeiten und Kosten im Vergleich zur } \\
\text { Zustellung allein mit konventionellen Vans (vor } \\
\text { allem bei hoher Kundendichte) sowie ggf. auch } \\
\text { Reduktion der gefahrenen Kilometer (aber auf- } \\
\text { grund geringer Ladekapazität ggf. mehr Fahrten/ } \\
\text { Fahrleistung erforderlich) }\end{array}$ & $\begin{array}{l}\text { Einschränkung der Bewegungsfreiheit aller Fuß- } \\
\text { gängerlnnen } \\
\text { - Gefährdung von FußgängerInnen, insbesondere } \\
\text { mobilitätseingeschränkten Personen, Kindern, } \\
\text { Älteren etc. } \\
\text { - Konfliktpotentiale beim Queren der Fahrbahn am } \\
\text { Fußgängerüberweg oder der Lichtsignalanlage, } \\
\text { beim Überholen von langsam gehenden Fußgän- } \\
\text { gerInnen oder beim Kontakt mit einem größeren } \\
\text { Pulk von Personen } \\
\text { - Sicherheitsaspekte (z. B. Diebstahl von Fahrzeu- } \\
\text { gen) und Vandalismus } \\
\text { Erfassung personenbezogener Daten }\end{array}$ \\
\hline
\end{tabular}




\section{PLANERISCHE IMPLIKATIONEN}

Erste Erfahrungen bei Tests mit automatisierten Zustellrobotern lassen wirtschaftliche Potentiale für die Distributionslogistik auf der letzten Meile erhoffen. Hier braucht es zukünftig aber noch weitere detaillierte und räumlich differenzierte Untersuchungen, in welchen Gebieten sich welche Potentiale für die Bewältigung der letzten Meile ergeben. Die Erfahrungen aus den Tests demonstrieren darüber hinaus jedoch auch die Probleme und Risiken von Zustellrobotern, wenn diese im öffentlichen Raum - insbesondere auf Gehwegen - unterwegs sind. Welche Aspekte für die Planung und (Verkehrs-)Politik zu beachten sind und welche Handlungsspielräume existieren, soll daher kurz diskutiert werden.

Schon heute besteht speziell im städtischen Raum - neben der Distribution von Waren - eine Vielzahl von weiteren Ansprüchen an den öffentlichen Straßenraum, die Nutzungs- und Interessenskonflikte implizieren (vgl. Buthe et al. 2018: 121). Zustellroboter erhöhen - neben Scootern, Ladezonen etc. - besonders in dichten urbanen Stadtquartieren zusätzlich den ohnehin schon hohen Nutzungsdruck auf den öffentlichen Raum - besonders auf den Gehwegen. So ist der öffentliche Raum nicht nur Verkehrsraum, sondern ebenso ein Ort für Aufenthalt und Begegnung (vgl. Stadt Wien 2018: 11), gerade im Übergang von Gebäude und Straßenraum auf den Gehwegen, wo sich Menschen unterhalten, Schaufenster betrachten etc. Der konkurrierende Flächenanspruch von Zustellrobotern, Gehwege zum Fahren und Abstellen zu nutzen, verursacht jedoch gerade in dichten urbanen Stadtquartieren Konflikte (vgl. Peters 2019: 76). Dies ist umso problematischer, als dass sich (1) gerade dort Lieferkonzepte mit Zustellrobotern aufgrund der hohen Kundendichte und damit Wirtschaftlichkeit per se besser eignen und (2) Zustellroboter, die vom Gehweg aus operieren, immer eine geringe Ladekapazität haben werden (Fahrzeugbreite kann kaum größer sein als der Bewegungsraum eines Menschen, Anforderungen an den automatisierten Umschlag vom Roboter in die Paketbox ermöglicht keine optimale Ausnutzung des Stauraums des Roboters, z. B. Einsortierung der Pakete in umgekehrter Reihenfolge der Auslieferung) und daher mit einem größeren Fahrzeugbedarf als beim Einsatz von konventionellen Auslieferungsfahrzeugen und auch im Vergleich zu Lastenrädern gerechnet werden kann. Dem steht auch der Anspruch der „Rückeroberung des öffentlichen Raums“ gegenüber, Verkehrsflächen zu reduzieren und Aufenthaltsflächen für mehr Lebensqualität und ein attraktives Wohnumfeld zu erhöhen. Auch schränken Lieferroboter kommerzieller Anbieter die Nutzung des öffentlichen Raums durch alle Menschen ein und tragen zu einer „Privatisierung öffentlicher Räume“ bei (vgl. Marks 2019: 14; Wong 2017): Der Gehweg gehört ganz klar den Fußgängerlnnen.

Wenn sich Zustellroboter durchsetzen, ist außerdem mit einem zusätzlichen Anpassungsbedarf der Infrastruktur und infolgedessen mit weiteren Kosten zu rechnen. So setzen Lieferkonzepte mit Zustellrobotern Paketboxen bei Haushalten voraus, da eine persönliche Zustellung an der Haustüre aufgrund der fehlenden Steigungsfähigkeit der Roboter kaum umsetzbar ist. Oder die Grünzeiten an Lichtsignalanlagen für Fußgängerlnnen sind anzupassen, falls die Geschwindigkeit der Zustellroboter unter derjenigen der FußgängerInnen liegt. Hier stellt sich die Frage, wer die Kosten übernimmt und wer letztlich auch für die Umsetzung verantwortlich ist (vgl. Hofer et al. 2018: 48).

Auch wenn die technologische Entwicklung derzeit noch nicht weit genug ist, dass der Zustellroboter im öffentlichen Raum auf Gehwegen zu jeder Zeit unter allen Bedingungen verkehren kann, ist es dennoch notwendig, jetzt den Zustellroboter „planerisch“ mitzudenken. Zum einen befinden wir uns gerade in einem Zeitalter der Transformation der öffentlichen Räume - weg vom Verkehrsraum hin zum Freiraum für alle -, zum anderen sind sehr frühzeitig die Qualitäten 
öffentlicher Räume zu sichern. Geschieht dies jetzt nicht, kann es passieren, dass ein Mix aus schnellen Technologiefortschritten bei den Zustellrobotern und starken Zunahmen von Lieferungen infolge von E-Commerce den politischen Handlungsdruck so schnell erhöhen, dass die nachfolgend erläuterten Regulative zu spät oder gar nicht greifen.

Auf der strategischen Ebene beeinflussen das Datenschutzrecht und das Straßenverkehrsrecht, die zur nationalen (bzw. EU-)Gesetzgebung zählen, den Einsatz, ob und wie Zustellroboter in öffentlichen Räumen eingesetzt werden können. Städte und Gemeinden können über ihre Verbände bzw. über das raumplanerische Gegenstromprinzip nationales Recht beeinflussen, haben aber auch eigene planerische Hebel. Dazu ist es notwendig, die Thematik Zustellrobotik in strategischen Konzepten mit Bezug zum öffentlichen Raum mit zu behandeln (z. B. Fachkonzept „Öffentlicher Raum“, Fachkonzept „Mobilität im Wiener Planungskontext“). Außerdem bedarf es innerhalb der Verwaltung, die Zuständigkeiten, Kompetenzen und Ressourcen dafür aufzubauen. Um abschätzen zu können, wo im urbanen Raum überhaupt Einsatzfelder liegen können, erscheinen kommunal initiierte Realexperimente, welche Nutzungskonflikte und Effekte der Zustellroboter betrachten, als ein erster wichtiger Schritt. Dabei ist wichtig, dass die Städte und Gemeinden „das Heft in die Hand nehmen“.

Konkrete verkehrsplanerische Maßnahmen, die Städte und Gemeinden zur Steuerung ergreifen können, sind beispielsweise die räumlich und zeitlich differenzierte Verordnung von Sperrzonen („geofencing“), wenn die Verträglichkeit von Zustellrobotern im öffentlichen Raum nicht gegeben ist. Eine weitere Maßnahme ist die Lizenzierung von Zustellrobotern in Stadtbereichen, um deren Anzahl zu beschränken und um Auflagen für den Betrieb erteilen zu können. Die Maßnahme der Echtzeitbepreisung stellt ein marktwirtschaftliches Instrument dar, das in Abhängigkeit der räumlich-zeitlichen Verträglichkeit der Zustellroboter im öffentlichen Raum dynamische Gebühren einhebt. Wie diese Maßnahmen konkret ausgestaltet und an räumliche Situationen angepasst werden sowie sich optimal ergänzen können, ist derzeit noch offen und zu erforschen.

\section{FAZIT}

Der E-Commerce gewinnt immer mehr Marktanteile, wodurch die Anzahl der Lieferungen zu den Haushalten rapide ansteigt. Logistikunternehmen profitieren durch die Automatisierung und Digitalisierung dann, wenn es gelingt, die Prozesse der "last mile“ - insbesondere der Kosten, aber auch Zustellzeiten - zu optimieren. Zustellroboter gelten hier als der große Hoffnungsträger. Auch wenn viele technologische Fragen derzeit noch ungelöst sind, so sind Zustellroboter schon heute in einigen Städten unterwegs und werden es zukünftig in weiteren Städten sein. Niedrige Geschwindigkeiten des Zustellroboters verknüpft mit einer einfachen Einsatzumgebung (ODD) sprechen eher für einen frühzeitigen Einsatz. In welchem Umfang auf Gehwegen einfache Einsatzumgebungen anzutreffen sind, ist mehr als offen. Während im Bereich der Fahrbahn klare Verkehrsregeln und Bewegungsrichtungen der Verkehrsteilnehmerlnnen existieren, gelten auf Gehwegen dynamische Regeln des Abstands- und Gehverhaltens: Fußgängerlnnen verändern Gehgeschwindigkeiten und Richtungen beliebig. Mit steigender Fußgängerdichte nehmen die Anforderungen an die Navigationsfähigkeit des Zustellroboters zu, sodass aus einfachen Einsatzumgebungen am Gehsteig sehr komplexe werden (vgl. Keesmaat 2020: 16) 
Abbildung 6: Überblick der unterschiedlichen Nutzungsansprüche und möglichen Konflikte im öffentlichen Raum unter Berücksichtigung von Zustellrobotern

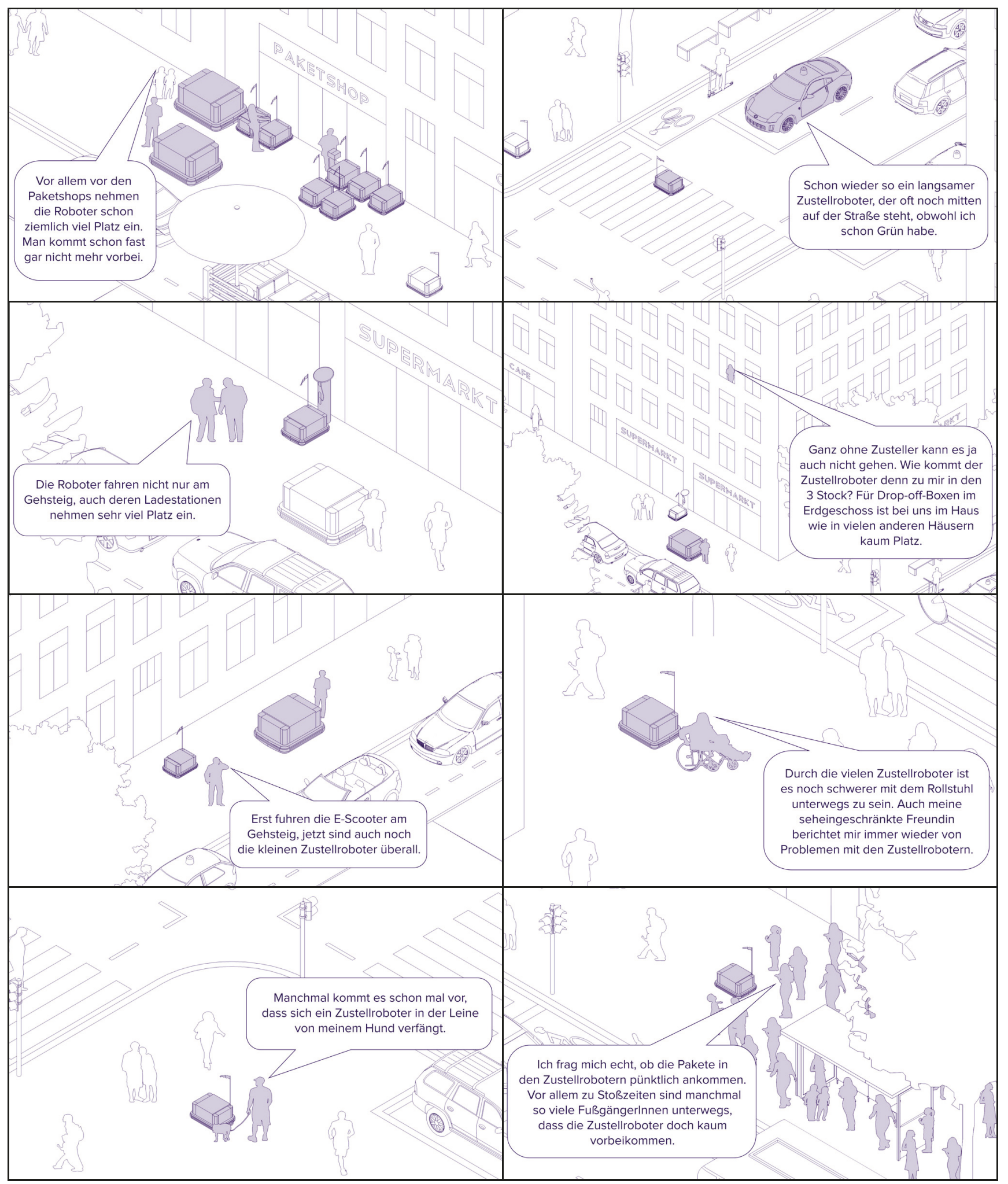

Quelle: eigene Darstellung 
Welche Einsatzszenarien der Zustellroboter sind denkbar? Ein mögliches und wahrscheinliches Szenario ist, dass sie im Bereich suburbaner, lockerer Bebauung, auf überbreiten Fahrbahnflächen mit wenig Verkehr eher konfliktarm unterwegs sein werden (da hier Themen wie beispielsweise die Vorbeifahrt an haltenden Fahrzeugen eine geringere Rolle spielen), wobei die wirtschaftliche Tragfähigkeit fraglich ist. Als verkehrsplanerische Maßnahmen zur Steuerung kommen beispielsweise eine Echtzeitbepreisung in Abhängigkeit der Fußgängerdichte und/ oder eine Regulation mittels Lizensierung in Frage.

Das andere Szenario, Zustellroboter in der dicht bebauten Stadt einzusetzen, ist mit wesentlich höheren Risiken und negativen Auswirkungen verbunden. Eine starke Regulierung über Sperrzonen, eine Lizenzierung etc. erscheinen notwendig. Der Gehsteig ist eigentlich tabu und wenn, dann sollten die Zustellroboter nur auf der Fahrbahn oder im Bereich des ruhenden Verkehrs fahren (Parkstreifen anders nutzen).

Aktuelle Pilotversuche zeigen jedoch, dass sich die Entwicklung auf Zustellroboter fokussiert, die auf Gehwegen unterwegs sind. Diese Entwicklung steht der Qualifizierung des öffentlichen Raums für mehr Aufenthalt und weniger Transit diametral entgegen. Die Gehsteige sind dabei besonders wichtige Orte des öffentlichen Raums, die als lineare Freiräume eine Vielzahl an Funktionen erfüllen: Sich kurz begegnen, rasten, warten, spielen, laufen, beobachten, schauen und sitzen sind für das urbane Alltagsleben elementar. Diese Qualitäten werden bereits heute oft unterschätzt, wie häufiges Gehsteigparken zeigt.

Zustellroboter - fahrend auf dem Gehweg bzw. abgestellt an Abhol-, Ladestationen oder auf dem Gehweg - verursachen mehr Nutzungsdruck, verschärfen die Flächenkonkurrenz und gefährden PassantInnen. Sie sind demnach dort besonders kritisch, wo es eng ist, wo viele Menschen unterwegs sind und betreffen besonders Personen mit Mobilitätseinschränkungen, Kinder und Ältere.

Die bisherigen Pilotversuche gehen zu wenig auf diese wichtigen Aspekte ein und stellen eindimensional die "drivability“ und Wirtschaftlichkeit der Zustellroboter in den Vordergrund. Der eigentliche Maßstab, ob ein Einsatz sinnvoll ist, muss sich an der Qualität des öffentlichen Raums orientieren. Hier besteht großer transdisziplinärer Experimentier- und Forschungsbedarf im Sinne der Reallabore, um der Komplexität des öffentlichen Raums gerecht zu werden. Zentral sind dabei die Fragen, welche und wie (straßen)räumliche, situative, soziale etc. Gegebenheiten die Akzeptanz der Zustellroboter und neuer Logistikkonzepte durch PassantInnen und Kundlnnen beeinflussen (vgl. Groot 2019: 64). Auch wie verkehrsplanerische Maßnahmen wirken könnten, ist dabei von besonderem Interesse, damit sich Städte und Gemeinden auf diese künftige Aufgabe vorbereiten können. Aktuell fehlen umfassende Evaluierungen zu den verkehrlichen Wirkungen und Umwelteffekten von Logistikkonzepten mit Zustellrobotern, zu den Effekten auf die Qualität öffentlicher Räume und zur Verkehrssicherheit, wobei sozialräumlich differenzierte Ansätze mit einem starken Nutzerfokus notwendig sind (Soteropoulos et al. 2019: 163). Hierbei geht es aber auch darum, die Gemeinwohlorientierung der Vehemenz der Interessensdurchsetzung kommerzieller AkteurInnen entgegenzusetzen und in demokratische Diskursprozesse zu bringen. 


\section{EIN INTERVIEW VON MARTIN BERGER UND AGGELOS SOTEROPOULOS MIT BERT LEERKAMP}

1. Welche Entwicklung im Bereich des E-Commerce ist aus Ihrer Sicht in den nächsten Jahren zu erwarten? Welche Wirkung hat die aktuelle Covid-19-Krise?

Bert Leerkamp: Der B2C-Versand nimmt nach verschiedenen Prognosen in den nächsten ca. 10 Jahren weiter mit relativ hohen Steigerungsraten (5 bis $10 \%$ pro Jahr) zu (Quellen und genaue Zahlen kann ich nachreichen). Hohe Wachstumsraten (auf zurzeit sehr geringem absolutem Niveau) zeigt der Onlinelebensmittelhandel. Im Extremfall (bei fehlender Durchreichung der Kosten an den Konsumenten) könnte dies zur teilweisen Aufhebung des „Pufferlagers Kühlschrank“ im Sinne einer verbreiteten On-Demand-Bestellung führen. Soweit ist es aber noch nicht. Andere Bereiche des Non-Food-Sektors scheinen ebenfalls noch überdurchschnittliches Nachfragepotential zu haben. Man muss allerdings zwischen dem Onlinevertrieb, zu dem auch „Click and Collect“ gehört (also Abholung durch den Kunden beim stationären Händler), und der Paketzustellung im B2C unterscheiden.

2. Güterverkehr in Städten war lange ein „unterbelichtetes“ Handlungsfeld in der kommunalen Verkehrs- und Stadtplanung. Ist das noch immer so? Wo liegen dabei die „großen“ verkehrspolitischen und planerischen Herausforderungen?

BL: In Deutschland hat die Klimaschutz- und Luftreinhaltedebatte und in der Folge die Bereitstellung diverser Förderprogramme seitens des Bundes und der Länder zu einer starken Aufmerksamkeitserhöhung und zu sehr vielen Aktivitäten in der Stadtlogistik geführt. Die große verkehrspolitische Herausforderung ist $m$. E. die Rückgewinnung der städtebaulichen bzw. stadtgestalterischen Qualität der öffentlichen Räume, die in vielen Städten im Zuge der autogerechten Stadt abhandengekommen ist und nun nur sehr mühsam und langsam wieder verbessert wird. Herausfordernd wird es sein, einen gesellschaftlichen Konsens für diesen Stadtumbau zu schaffen, der über die Beseitigung der Probleme und Handlungserfordernisse hinausgeht, die die aktuelle Luftschadstoffdebatte umfasst, und der mit dem Begriff einer umfassenden Verkehrswende beschrieben wird. Möglicherweise trägt das Klimaschutzargument nicht genug und nicht dauerhaft genug, um diese Verkehrswende umzusetzen. Vielleicht werden aber auch andere europäische Metropolen vorangehen und damit sehr wirksame Wettbewerbsvorteile bei der Ansiedlung technologieorientierter Unternehmen bekommen, die um hochqualifizierte Arbeitskräfte werben. Das könnte einen Schub für den Stadtumbau geben, der mit der Änderung von Mobilitätsverhalten einhergehen muss.

Die Logistik der städtischen Ver- und Entsorgung steht m. E. infolge einer solchen Verkehrswende gar nicht vor ganz großen Herausforderungen. Logistik ist daran gewöhnt, unter gegebenen Bedingungen optimale Lösungen zu finden und sich laufend selbst zu optimieren. Das heißt umgekehrt, dass die Städte diese Bedingungen eindeutig, klar und verlässlich definieren müssen. Ein Beispiel: Veränderungen der Zugänglichkeit der Innenstädte für den Lieferverkehr, wie wir sie in dem aktuellen Leitfaden „Liefern ohne Lasten“ (Herausgeber: Agora Verkehrswende 2020) beschrieben haben, müssen angekündigt und mit einer ausreichenden Vorlaufzeit verbindlich eingeführt werden. Im Austausch zwischen kommunaler Planung, Handel, Dienstleistungen und Transportwirtschaft muss komplementär dafür gesorgt werden, dass keine kontraproduktiven Maßnahmen geplant werden. 
3. Warum ist die „last mile“ der Zustellung für die Logistikunternehmen so kostenintensiv? Verändert sich dieser Kostenfaktor über die Jahre? Welche Trends beeinflussen die Kostendynamiken? Wie groß ist hier die Motivation der Logistikunternehmen, Aufwände einzusparen?

BL: Folgende Effekte haben zum Beispiel (dabei handelt es sich hier um keine vollständige Auflistung) zu Kostensteigerungen beigetragen bzw. werden in Zukunft kostensteigernd wirksam:

1. kontinuierlich zunehmende Entfernung zwischen den letzten Umschlagpunkten (Speditions- und KEP-Depots) und den Lieferzielen, die längere Fahrtwege und damit auch einen größeren Personal- und Fahrzeugeinsatz bewirken (Auslagerung der Logistikstandorte aus den Kernbereichen der Städte) und

2. zunehmende Serviceanforderungen, insbesondere Terminlieferungen, die die Bündelungsfähigkeit von Sendungen reduzieren.

3. Diversifizierung des Warenangebotes im Konsumgüterbereich und gleichzeitig aufkommende Konkurrenz durch den Onlinehandel haben dazu geführt, dass der Handel seine Reaktionsfähigkeit erhöhen musste und weiter erhöhen muss (damit sind häufigere Anlieferungen von mehr Versendern mit kleineren Sendungsgrößen verbunden, also auch hier negative Auswirkungen auf die Bündelungsfähigkeit).

4. Im Onlinehandel wirken Terminsendungen ebenfalls kostentreibend: gegebene und gut begründete Lieferzeitfenster in den Städten führen dazu, dass die KEP-Dienstleister im kurzen morgendlichen Auslieferungszeitfenster (welches durch Tendenz zu späterer Ladenöffnung weiter verkürzt wird) mit mehreren Auslieferungsfahrzeugen gleichzeitig in die City fahren müssen, um alle Sendungen abliefern zu können. Danach werden die restlichen Sendungen im weiteren Stadtgebiet verteilt (die gebietsbezogene Bündelungsfähigkeit wird dadurch reduziert).

5. Weiteres KEP-Mengenwachstum im B2C überbeansprucht die Kapazitäten der Logistiker, sodass „Negative economies of scale“-Effekte eintreten (zusätzliches Aufkommen erzeugt überproportional steigende Handlungskosten bei nicht steigendem Erlös je Sendung).

6. Kostenreduktionspotentiale (auch durch Lohndumping und Outsourcing an Subunternehmer) sind ausgereizt und können die Kostensteigerungen nicht mehr dämpfen. Zukünftig werden die Löhne steigen, weil die Arbeitskräfte fehlen. Auch die Beschaffung von E-Fahrzeugen infolge der Einführung von Nullemissionszonen wird zu höheren Kosten führen.

4. Wie schätzen Sie die technologische Entwicklung der Zustellroboter ein? Wo liegt der aktuelle Fokus der Forschung? Gibt es große Fortschritte? Wo liegen die größten technologischen Hürden für einen wirtschaftlichen Einsatz in der Praxis? Welcher Entwicklungspfad ist wahrscheinlich - eher fahrbahn- oder gehwegtauglich?

BL: Die mir bekannten Vorhaben erscheinen mir oftmals als Demonstrationsvorhaben mit einem hohen Anteil an Marketingzielsetzungen - Unternehmen wollen sich als innovativ präsentieren und darstellen, dass sie Teil der Lösung bestehender Probleme sind. Eine unabhängige und umfassende (ganzheitliche) Technikfolgenabschätzung vermag ich noch nicht zu erkennen. Die regulatorischen Rahmensetzungen werden m. E. oft nicht beleuchtet, es geht um technische Machbarkeit und es soll der Eindruck vermittelt werden, die autonomen Fahrzeuge könnten einen großen Teil des Zustellbetriebs übernehmen. Positive Umwelteffekte werden z. T. falsch zugeordnet bzw. es wird unterstellt, sie seien ein 
Spezifikum der autonomen Fahrzeuge: Tatsächlich sind die Effekte aber dem E-Antrieb zuzuordnen und der Vergleich zu anderen Lösungsansätzen wie z. B. dem Lastenrad fehlt.

Die autonomen bzw. automatisierten technischen Systeme müssen unter "chaotischen“ Bedingungen zuverlässig funktionieren, $d$. h. es kommt im Praxiseinsatz zu vielfältigsten Kombinationen einzelner Störungen. Menschen finden in solchen Situationen schnell akzeptable Lösungen. Dem technischen System müssen alle möglichen Störungen/Störungskombinationen einprogrammiert werden, d. h. es müssen dafür Lösungen vorbereitet werden. Zusätzlich müssen praxistaugliche Systeme Redundanzen in den sicherheitstechnisch relevanten Systemteilen aufweisen. Dies alles reduziert die Wirtschaftlichkeit der Systeme und auch ihre Anfälligkeit gegen den Ausfall von Technik (Sensorik, Mechanik, Energieversorgung ...). Der Überwachungs- und Wartungsaufwand sowie Eingriffe bei Störungen erzeugen zusätzliche Kosten.

Im Labor herstellbare Bedingungen der Systemumgebung (hier: des öffentlichen Raums) sind in der Realität oft nicht umsetzbar. Ich halte es etwa für schwierig machbar, einen Zustellroboter nur auf dem Gehweg und nicht auf dem angrenzenden Radweg zu navigieren, wenn dieser nicht sehr gut erkennbar ist - welche Sensorik leistet das? Forderungen an eine der Technik entsprechenden Gestaltung öffentlicher Räume wären fragwürdig - wer trägt die Kosten, wer hat den Nutzen? - und praktisch nicht flächendeckend umsetzbar.

Im Bereich der „Massenverkehre“, also der Paketzustellung in Verdichtungsräumen, wird die Zustellung durch Menschen weiter die Regel bleiben, weil sie wirtschaftlicher ist. In sehr dünn besiedelten Räumen kann ich mir automatisierte bzw. autonome Systeme (land- oder luftgestützt) in Sondersituationen vorstellen (u. a. Belieferung von Inseln mit eiligen Gütern per Drohne, Zustellung an Einzelgehöfte/Almen in bergigen Gegenden ...). Die Nischeneinsätze (wie oben erwähnt) werden eher die Fahrbahn als den Gehweg betreffen, weil es dort keine Gehwege gibt.

\section{Konflikte zwischen Zustellrobotern und Menschen im Straßenraum sind} vorprogrammiert. Wo liegen die „Knackpunkte“? Was sind die Akzeptanzprobleme? Ist aus Ihrer Sicht der Gehsteig „Tabu“ für den Zustellroboter? Gibt es Gebiete, die sich aus Ihrer Sicht eher für Zustellroboter eignen und welche besonders problematisch sind? Was sind die Einsatzkriterien?

BL: Menschen werden Behinderungen durch technische Systeme nicht akzeptieren, weil dies als ungerechte Verteilung von individuellem Nutzen (der Belieferten, der Logistiker) und kollektive Belastung empfunden werden wird. Gehwege an typischen Hauptverkehrsstraßen sind heute schon zu schmal und führen zu hohem Interaktionsbedarf zwischen Menschen. Wenn technische Systeme interagieren sollen, kann die Lösung vermutlich nur lauten, dass diese dem Menschen ausweichen, was aber den Betrieb der autonomen Systeme beeinträchtigen wird.

Praktisch ist der Gehweg aus den o. g. Gründen tabu und gleichzeitig aus technischer Sicht weithin ungeeignet. Einsatzkriterien sind

- die uneingeschränkte Kompatibilität zur heutigen Welt (kein Anpassungsbedarf der Systemumgebung an das System) sowie keine Behinderungen des Fuß- und Radverkehrs, mobilitätseingeschränkter, seh- und hörbehinderter Menschen,

- betriebswirtschaftliche Vorteile unter o. g. Rahmenbedingungen,

- $\quad$ keine verkehrssicherheitsrelevanten negativen Wirkungen. 
6. Wenn E-Commerce immer mehr Marktanteile gewinnt und der stationäre Einzelhandel verliert, entfallen auch Einkäufe zu Fuß. Wie schätzen Sie diese Verlagerungen von physischer zu virtueller Mobilität ein? Sind dadurch weniger FußgängerInnen auf den Gehsteigen unterwegs, die den Zustellroboter „stören“ können und dessen Verbreitung zusätzlich fördern?

BL: Aus Mobilitätserhebungen kann man abschätzen, welcher Anteil des Fußverkehrs dem Wegezweck Einkauf dient. In Geschäftsbereichen konzentriert sich das zwar, bleibt aber nur ein mitunter kleiner Teil, von dem ggf. ein wenig auf den Onlinehandel verlagert wird. Die Menschen werden aber in der gewonnenen Zeit (in der sie nicht einkaufen gehen müssen) vielleicht ins Café gehen und also auch Fußwege zurücklegen. Also insgesamt: marginale, eher theoretische Effekte. Und wollen wir menschenleere Straßen?

7. Bereits seit Jahren wird angestrebt, FußgängerInnen und RadfahrerInnen mehr Platz im öffentlichen Raum zur Verfügung zu stellen (Umverteilung von Flächen im öffentlichen Raum) - bislang jedoch nur mit mäßigem Erfolg. Im Zuge der Covid-19-Krise finden jedoch in zahlreichen Städten wie beispielsweise Berlin, Wien oder Brüssel temporäre Umnutzungen von Fahrbahnen oder Parkplätzen für FußgängerInnen oder RadfahrerInnen statt. Kann diese Krise hier als Tipping-Point gesehen und als Chance genutzt werden, tatsächlich eine Umverteilung von Flächen im öffentlichen Raum zu erreichen?

BL: Ich beobachte, dass Kommunen sehr zögerlich und wenig spontan auf diese Chance zugehen. Berlin ist eine Ausnahme. Oft wird auf bestehende Konzepte sowie auf fehlende Arbeitsaufträge durch den Rat der Stadt verwiesen - damit fehle die Handlungsgrundlage. Gleichzeitig nehme ich wahr, dass die Umverteilungsfrage immer häufiger von mehr Bevölkerungsgruppen und nachdrücklicher gestellt wird. Das sollte auch ohne Corona hoffentlich tragfähig werden und die politische Entscheidungsfindung beeinflussen.

8. Gemeinden und Städte sind wichtige Akteure, wenn es um die lokale Umsetzung neuer Mobilitätslösungen wie den Zustellrobotern geht. Welche Rahmenbedingungen auf den anderen politischen Ebenen sind notwendig? Gibt es in der lokalen Politik und Verwaltung eine Problemwahrnehmung bzw. einen Diskurs dazu? Welche Akteure setzen das Thema? Was sind deren Interessen und Motive? Wie nehmen Sie die Vehemenz der Interessensdurchsetzung kommerzieller Akteure wahr?

BL: In Deutschland ist die Straßenverkehrsordnung maßgebend, die vom Bund im Vernehmen mit den Ländern gestaltet wird. Die gerade erst eingeführte Elektrokleinstfahrzeugeverordnung des Bundes wäre anzupassen. In der lokalen und bundesweiten Politik nehme ich wahr, dass die hier diskutierten Konzepte (Zustellroboter) oft recht undifferenziert als innovative Lösungen angesehen werden, die grundsätzlich positiv konnotiert sind. Teilweise kommt es mir so vor, dass man mit einer Fokussierung auf und der undifferenzierten Begrüßung von autonomen Zustellsystemen von Handlungsbedarfen ablenken will, die nicht in die eigenen Zielvorstellungen passen und unbequemer durchzusetzen wären (Technologieprimat gegenüber dem integralen Ansatz der Verkehrswende).

Kommerzielle Akteure der Logistik gehen sehr nüchtern mit dem Thema um und sehen nach meiner Einschätzung kaum Potenziale. Jüngst hat in einem AK Urbane Logistik in Düsseldorf keiner der anwesenden KEP-Unternehmen Zustellroboter als Lösungsansatz erwähnt.

9. Macht es Sinn, dass die Gemeinden und Städte bereits jetzt handeln und die Nutzung öffentlicher Räume durch Zustellroboter regulieren oder sind die Umstände 
noch viel zu unsicher? Geht die Entwicklung der Regulative „Hand in Hand“ mit dem technologischen Fortschritt? Welche regulatorischen Neuerungen werden diskutiert und wie sehen Sie die Chancen und Risiken, dass diese ergriffen werden? In San Francisco beispielsweise werden für den Betrieb von Zustellrobotern Lizenzen für einzelne Anbieter ausgegeben. Ist ein solches Modell auch für Gemeinden und Städte im deutschsprachigen Raum denkbar?

BL: Ein frühzeitiges Signal der Kommunen zu ihren Präferenzen der Nutzung von Gehwegen mag für diejenigen hilfreich sein, die das Thema rein technisch betrachten und problematische Rahmenbedingungen ausblenden. Ich habe keinen Überblick, wo derzeit welche regulatorischen Neuerungen diskutiert werden. Mit lizenzierten Sharingsystemen (E-Roller) machen Kommunen in Deutschland zurzeit ambivalente Erfahrungen. Der Regelungs- und Nachsteuerungsbedarf ist hoch (u. a. verbotene Bereiche/Flächen), die im kommunalen Interesse liegenden Beiträge zu einem nachhaltigen Verkehr stehen im Konflikt mit beobachtbaren negativen Auswirkungen ( $u$. a. verbreitetes Liegenlassen von E-Rollern auf Gehwegen, Nutzung als Freizeitspaß in der Nacht mit Ruhestörung etc.). Die Kommunen müssten sich hier in ein neues Rechtsgebiet einarbeiten. Grundsätzlich zu klären wäre das Verhältnis von Grundrecht auf allgemeine und uneingeschränkte Nutzung des öffentlichen Raums versus Lizenzierung (d. h. Nutzungsbeschränkung).

10. Welche Alternativen zur „Bewältigung“ der letzten Meile gibt es abseits der Zustellroboter? Gibt es andere technologische Entwicklungen mit Potential - z. B. Drohnen? Oder braucht es soziale bzw. organisatorische Innovationen? Bedarf es Verhaltensänderungen der Kunden oder reichen technische bzw. infrastrukturelle Maßnahmen aus, um die Qualität im öffentlichen Raum zu sichern? Könnten hierbei auch Ansätze des Nudgings, also der Verhaltensbeeinflussung (z. B. Darstellung des $\mathrm{CO}_{2}$-Verbrauchs bei unterschiedlichen Lieferoptionen) sinnvoll sein?

BL: Zwei Optionen sehe ich vorrangig: (1) KEP-Dienstleister arbeiten mit Mikrohubs und Zustellung per Lastenrad von diesen Hubs aus und (2) es erfolgt eine gebietsbezogene Sendungsbündelung durch Empfangsspediteure: Diese fungieren als Zustelladresse der Versender, d. h. sind die Lieferadresse für die KEP-Dienstleister. Der Empfangsspediteur bündelt dann empfängerbezogen und liefert aus. Das System wird von der Firma $A B C$-Logistik in Düsseldorf mit rd. 150 Einzelhändlern erfolgreich betrieben und bündelt B2B-Paketsendungen des Handels und großer Bürostandorte. Dabei kommt auch ein Lastenrad zum Einsatz.

11. Wo liegt aus Ihrer Sicht der Forschungsbedarf an der Schnittstelle „Öffentlicher Raum und Last-Mile-Logistik mit oder ohne Zustellroboter"? Soll die öffentliche Hand Innovationen - wie den Zustellroboter - steuern oder eher auf den Markt setzen?

BL: Aktuell sehe ich Bedarf an einer transformativen Forschung, die Wandel unterstützt bzw. möglich macht, gute Lösungen testet und evaluiert und dann verbreitet. Den Bedarf, dass die öffentliche Hand Forschung initiiert, um den Zustellrobotereinsatz zu fördern, sehe ich nicht, da ich das wirtschaftliche Einsatzpotential in Städten und die gesellschaftlich positiven Effekte für gering halte. 


\section{LITERATUR}

APA 2017. „Österreich-Premiere: Autonomes E- Fahrzeug stellt Pakete im Alleingang zu!“. www.ots.at/ presseaussendung/OTS_20171018_OTS0033/oesterreich-premiere-autonomes-e-fahrzeug-stelltpakete-im-alleingang-zu (4.9.2020).

Baum, L., T. Assmann und H. Strubelt 2019. „State of the art - Automated micro-vehicles for urban logistics“, in IFAC-PapersOnLine (52) 13, 2455-2462.

BIEK (Bundesverband Paket und Expresslogistik) 2020. „KEP-Studie 2020 - Analyse des Marktes in Deutschland“. Köln. Download unter www.biek.de/download.html?getfile=2623 (26.8.2020).

Brandt, C., B. Böker, A. Bullinger, M. Conrads, A. Duisberg und S. Stahl-Rolf 2018. „Fallstudie: Delivery Robot Hamburg für KEP-Zustellung", im Auftrag des Bundesministeriums für Wirtschaft und Energie (BMWi), Berlin/Düsseldorf: VDI Technologiezentrum. www.bmwi.de/Redaktion/DE/Downloads/C-D/delivery-robot-hamburg.pdf?__blob=publicationFile\&v=4 (27.8.2020).

Buchholz, K. 2019. „87 Billion Parcels Were Shipped in 2018“, Statista, 8.11.2019. www.statista.com/ chart/10922/parcel-shipping-volume-and-parcel-spend-in-selected-countries/ (26.8.2020).

Buthe, B., J. Modes, B. Richter, H.-P. Kienzler, S. Altenburg, K. Esser, J. Kurter, D. Wittowsky, K. Konrad, A.-L. van der Vlugt und S. Groth 2018. „Verkehrlich-Städtebauliche Auswirkungen des Online-Handels“, Endbericht, 16.8.2018. Bonn: Bundesinstitut für Bau-, Stadt- und Raumforschung. www.prognos.com/uploads/tx_atwpubdb/180927_BBSR_Endbericht_final_out.pdf (26.8.2020).

Clausen, U. 2017. „Was tut sich auf der letzten Meile?“, in Handelsblatt Journal 5, Sonderveröffentlichung zum Thema „Die Zukunft der Automobilindustrie“, $16 \mathrm{f}$.

Daimler 2019. „Daimler Trucks investiert eine halbe Milliarde Euro in hochautomatisierte Lkw“. https:// media.daimler.com/marsMediaSite/de/instance/ko/Daimler-Trucks-investiert-eine-halbe-MilliardeEuro-in-hochautomatisierte-Lkw.xhtml?oid=42188247 (19.8.2020).

Deutsche Bundesregierung 2019. „Projektionsbericht 2019 für Deutschland gemäß Verordnung (EU) Nr. 525/2013“. Berlin. https://cdr.eionet.europa.eu/de/eu/mmr/art04-13-14_Icds_pams_projections/projections/envxnw7wq/Projektionsbericht-der-Bundesregierung-2019.pdf (19.8.2020).

DHL 2014. „Self-driving vehicles in Logistics. A DHL Persperctive on implication and use cases for the logistics industry“. Troisdorf. https://discover.dhl.com/content/dam/dhl/downloads/interim/full/dhlself-driving-vehicles.pdf (27.8.2020).

Eigner, S. 2017. „Post und TU Graz erproben autonome Transportlogistik auf der ,letzten Meile““. www. tugraz.at/tu-graz/services/news-stories/medienservice/einzelansicht/article/post-ag-und-tu-grazerproben-autonome-transportlogistik-auf-der-letzten-meile/ (4.9.2020).

ERTRAC (European Road Transport Research Advisory Council) 2014. „Urban Freight research roadmap“. Brüssel. www.ertrac.org/uploads/documentsearch/id36/ERTRAC_Alice_Urban_Freight.pdf (7.1.2020).

Flämig, H. 2015. „Autonome Fahrzeuge und autonomes Fahren im Bereich des Gütertransportes“, in Autonomes Fahren. Technische, rechtliche und gesellschaftliche Aspekte, hg. v. M. Maurer, J. C. Gerdes, B. Lenz und H. Winner. Berlin/Heidelberg: Springer Vieweg, 377-398.

Gerdes, J., und G. Heinemann 2019. „Urbane Logistik der Zukunft - ganzheitlich, nachhaltig und effizient", in Handel mit Mehrwert. Digitaler Wandel in Märkten, Geschäftsmodellen und Geschäftssystemen, hg. v. G. Heinemann, H. M. Gehrckens und T. Täuber, Wiesbaden: Gabler, 397-420.

Groot, S. de 2019. „Pedestrian Acceptance of Delivery Robots: Appearance, interaction and intelligence design“, Masterarbeit, TU Delft.

Heinemann, G., H. M. Gehrckens und T. Täuber 2019. Handel mit Mehrwert. Digitaler Wandel in Märkten, Geschäftsmodellen und Geschäftssystemen. Wiesbaden: Gabler.

Hermes 2017a. „,Wir haben etwas Neues auf den Fußweg gebracht‘: Erste Learnings aus dem Pilottest mit unserem Zustellroboter", Hermes bloggt, 31.3.2017. https://blog.myhermes.de/2017/03/wir-haben-etwas-neues-auf-den-fussweg-gebracht-erste-learnings-aus-dem-pilottest-mit-unserem-zustellroboter/ (8.1.2020).

Hermes 2017b. „Starship-Roboter in Hamburg: ,Innovation passiert nicht am Schreibtisch““, Hermes Newsroom, 29.3.2017. https://newsroom.hermesworld.com/starship-roboter-in-hamburg-innovation-passiert-nicht-am-schreibtisch-12146/ (7.1.2020). 
Hern, A. 2018. „First robot delivery drivers start work at Silicon Valley campus“, The Guardian, 30.4.2018. www.theguardian.com/cities/2018/apr/30/robot-delivery-drivers-coming-to-a-campus-near-youstarship-technologies (7.1.2020).

Hörl, S., F. Becker, T. Dubernet und K. W. Axhausen 2019. „Induzierter Verkehr durch autonome Fahrzeuge: Eine Abschätzung“, Forschungsprojekt SVI 2016/001 auf Antrag der Schweizerischen Vereinigung der Verkehrsingenieure und Verkehrsexperten (SVI). Bern: Eidgenössisches Departement für Umwelt, Verkehr, Energie und Kommunikation UVEK. https://ethz.ch/content/dam/ethz/ special-interest/baug/ivt/ivt-dam/vpl/reports/1401-1500/ab1433.pdf (18.8.2020).

Hofer, M., L. Raymann und F. Perret 2018. „Einsatz automatisierter Fahrzeuge im Alltag - Denkbare Anwendungen und Effekte in der Schweiz. Schlussbericht Modul 3f ,Güterverkehr / City Logistik (Strasse)“", definitive Fassung vom 28.3.2018. Zürich: EBP Schweiz. www.ebp.ch/sites/default/ files/project/uploads/2018-03-28\%20aFn_3f\%20Güterverkehr\%20und\%20Citylogistik_Schlussbericht_0.pdf (27.8.2020).

Hoffmann, T., und G. Prause 2018. „On the Regulatory Framework for Last-Mile Delivery Robots“, in Machines (6) 3, 1-16.

Howell, A., H. Tan, A. Brown, M. Schlossberg, J. Karlin-Resnick, R. Lewis, M. Anderson, N. Larco, G. Tierney, J. Carlton, J. Kim und B. Steckler 2020. „Multilevel Impacts of Emerging Technologies on City Form and Development“. Eugene, OR: Urbanism Next Center, University of Oregon. Download unter https://scholarsbank.uoregon.edu/xmlui/bitstream/handle/1794/25191/R_NSF_Multilevellmpacts.pdf?sequence=1\&isAllowed=y (27.8.2020).

Hsu, J. 2019. „Out of the Way, Human! Delivery Robots Want a Share of Your Sidewalk“, Scientific American, 19.2.2019. www.scientificamerican.com/article/out-of-the-way-human-delivery-robots-wanta-share-of-your-sidewalk/ (27.8.2020).

Jennings, D., und M. A. Figliozzi 2019. „Study of Sidewalk Autonomous Delivery Robots and Their Potential Impacts on Freight Efficiency and Travel“, in Transportation Research Record (2673) 6, 317-326.

Just, M. 2018. „Lieferservice und Güterverkehr in der Stadt - Historie, zukünftige Entwicklungen und Lösungsmöglichkeiten“, Vortrag, 25.1.2018. München. https://docplayer.org/73063319-Lieferservice-und-gueterverkehr-in-der-stadt-historie-zukuenftige-entwicklungen-und-loesungsmoeglichkeiten.html (26.8.2020).

Keesmaat, P. 2020. „Designing socially adaptive behavior for mobile robots“, Masterarbeit, TU Delft.

Knoppe, M., und M. Wild 2018. Digitalisierung im Handel. Geschäftsmodelle, Trends und Best Practice. Wiesbaden: Gabler.

Kunze, O. 2016. „Replicators, Ground Drones and Crowd Logistics A Vision of Urban Logistics in the Year 2030“, in Transportation Research Procedia 19, 286-299.

Leerkamp, B. 2017. „Städtische Güterverkehrskonzepte - die erste und die letzte Meile“, Vortrag, Zukunftsfähige Mobilität in Wuppertal - Handel und Verkehr, 23.5.2017. https://docplayer.org/61079802-Staedtische-gueterverkehrskonzepte-die-erste-und-die-letzte-meile.html (26.8.2020).

Leerkamp, B. 2019. „Beiträge der Raumplanung zum Klimaschutz im Güterverkehr“; in Nachrichten $\operatorname{der} A R L$ 1, 20-23. https://shop.arl-net.de/media/direct/pdf/nachrichten/2019-1/nr_1-19_leerkamp. pdf (26.8.2020).

Leitner, K.-H., T. Bacher, S. Humpl, A. Kasztler, A. Millonig, W. Rhomber und P. Wagner 2018. „Berufsbilder und Chancen für die Beschäftigung in einem automatisierten und digitalisierten Mobilitätssektor 2040“. Wien: Bundesministerium für Verkehr, Innovation und Technologie. https://mobilitaetderzukunft.at/resources/pdf/projektberichte/Mob_2040_Endbericht_2018_Septemberfinal.pdf (12.3.2020).

Leitschuh, V. 2018. „Zusteller der Zukunft: Lieferroboter in neuem Auftrag unterwegs“, Eimsbütteler Nachrichten, 10.1.2018. www.eimsbuetteler-nachrichten.de/lieferroboter-wieder-in-eimsbuettelunterwegs/ (7.1.2020).

Lenthang, M. 2019. „Autonomous food delivery robots that are just knee-high and travel on the sidewalk on college campuses are branded a menace for disabled people“, Daily Mail online, 20.11.2019. www.dailymail.co.uk/news/article-7706813/Self-driving-delivery-robots-college-campuses-menace-disabled-people.html (9.1.2020).

Lierow, M., und D. Wisotzky 2019. „Letzte Meile macht E-Food zu schaffen. Zustellung fordert Lebensmittel-Onlinehandel heraus - Alternative Lieferkonzepte gefragt“. www.oliverwyman.de/our-expertise/insights/2019/may/Letzte-Meile-macht-E-Food-zu-schaffen.html (19.8.2020). 
Marks, M. 2019. „Robots in Space: Sharing our World with Autonomous Delivery Vehicles“. https:// robots.law.miami.edu/2019/wp-content/uploads/2019/04/Marks_Robots-in-Space.pdf (7.1.2020).

Marazzo, A. und J. Mischler 2018. „Abschlussbericht Lieferroboter - Testphase 2“. Bern: Post CH AG. Download unter: www.astra.admin.ch/dam/astra/fr/dokumente/abteilung_strassennetzeallgemein/abschlussbericht_lieferrobotter.pdf.download.pdf/2018-08-23_\%20Post\%20_\%20Abschlussbericht\%20-\%20Lieferroboter\%20I1\%20.pdf (4.9.2020).

Maurer, M., J. C. Gerdes, B. Lenz und H. Winner 2015. Autonomes Fahren. Technische, rechtliche und gesellschaftliche Aspekte. Berlin/Heidelberg: Springer Vieweg.

Mitteregger, M., E. M. Bruck, A. Soteropoulos, A. Stickler, M. Berger, J. S. Dangschat, R. Scheuvens und I. Banerjee 2020. AVENUE21. Automatisierter und vernetzter Verkehr: Entwicklungen des urbanen Europa. Wiesbaden: Springer Vieweg.

Muschkiet, M., und U. Schückhaus 2019. „Anforderungen an die Handelslogistik der Zukunft“, in Handel mit Mehrwert. Digitaler Wandel in Märkten, Geschäftsmodellen und Geschäftssystemen, hg. v. G. Heinemann, H. M. Gehrckens und T. Täuber. Wiesbaden: Gabler, 357-378.

Ninnemann, J., A.-K. Hölter, W. Beecken, R. Thyssen und T. Tesch 2017. „Last-Mile-Logistics Hamburg Innerstädtische Zustelllogistik. Studie im Auftrag der Behörde für Wirtschaft, Verkehr und Innovation der Freien und Hansestadt Hamburg". Hamburg: HSBA Hamburg School of Business Administration. www.hsba.de/fileadmin/user_upload/bereiche/forschung/Forschungsprojekte/Abschlussbericht_Last_Mile_Logistics.pdf (7.1.2019).

Nuro 2018. „Scottsdale, meet Nuro“, 16.8.2018. https://medium.com/nuro/az-pilot-launch-33cceb55c871 (27.8.2020).

Nuro 2020. „Nuro. FAQ. How will I retrieve my order? Who can participate in Nuro's service?“. https:// nuro.ai/faq (28.8.2020).

open4innovation 2019. „, Nachhaltige Lösungen für die First / Last-Mile: Innovative Logistiklösungen für Zustellung, Auslieferung und Abholung“. Wien: Bundesministerium für Klimaschutz, Umwelt, Energie, Mobilität, Innovation und Technologie (BMK). https://mobilitaetderzukunft.at/de/highlights/ first-last-mile.php (4.9.2020).

Paddeu, D., T. Calvert, B. Clark und G. Parkhurst 2019. „New Technology and Automation in Freight Transport and Handling Systems“. Download unter https:/uwe-repository.worktribe.com/output/851875/ new-technology-and-automation-in-freight-transport-and-handling-systems (27.8.2020).

Peters, N. 2019. „Die Letzte Meile im urbanen Güterverkehr und ihre Auswirkungen auf öffentliche Räume“, Diplomarbeit, TU Wien.

Schnedlitz, P., E. Lienbacher, B. Waldegg-Lindl und M. Waldegg-Lindl 2013. „Last Mile: Die letzten und teuersten - Meter zum Kunden im B2C E-Commerce“, in Handel in Theorie und Praxis. Festschrift zum 60. Geburtstag von Prof. Dr. Dirk Möhlenbruch, hg. v. G. Crockford, F. Ritschel und U.-M. Schmieder. Wiesbaden: Gabler, 249-273.

Schocke, K.-O. 2019. „Boom mit Problemen: Paketbranche feilt an ,letzter Meile““, Die Zeit, 6.3.2019. www.zeit.de/news/2019-03/06/boom-mit-problemen-paketbranche-feilt-an-letzter-meile-190306 -99-264826 (26.8.2020).

Schönberg, T., T. Wunder und M. S. Huster 2018. „Urbane Logistik 2030 in Deutschland - Gemeinsam gegen den Wilden Westen“, hg. v. Roland Berger. München. Download unter https://www.bvl.de/ schriften/schriften/urbane-logistik-2030 (26.8.2020).

Schröder, J., B. Heid, F. Neuhaus, M. Kässer, C. Klink und S. Tatomir 2018. „Fast forwarding last-mile delivery - implications for the ecosystem: Travel, Transport, and Logistics and Advanced Industries“, hg. v. McKinsey \& Company. www.mckinsey.com/de/ /media/mckinsey/locations/europe\%20 and\%20middle\%20east/deutschland/publikationen/fast\%20forwarding\%20last\%20mile/180712fast-forwarding-last-mile-delivery.ashx (27.8.2020).

Shaheen, S., und A. Cohen 2020. „Mobility on Demand in the United States. From Operational Concepts and Definitions to Early Pilot Projects and Future Automation“, in Analytics for the Sharing Economy: Mathematics, Engineering and Business Perspectives, hg. v. E. Crisostomi, B. Ghaddar, F. Häusler, J. Naoum-Sawaya, G. Russo und R. Shorten. Cham: Springer International Publishing, 227-254.

Soteropoulos, A., A. Stickler, V. Sodl, M. Berger, J. Dangschat, P. Pfaffenbichler, G. Emberger, E. Frankus, R. Braun, F. Schneider, S. Kaiser, H. Walkobinger und A. Mayerthaler 2019. „SAFiP - Systemszenarien Automatisiertes Fahren in der Personenmobilität“. Wien: Bundesministerium für Verkehr, Innovation und Technologie. https://projekte.ffg.at/anhang/5cee1b11a1eb7_SAFiP_Ergebnisbericht. pdf (27.8.2020). 
Soteropoulos, A., M. Mitteregger, M. Berger und J. Zwirchmayr 2020. „Automated drivability: Toward an assessment of the spatial deployment of level 4 automated vehicles", in Transportation Research Part A: Policy and Practice 136, 64-84.

Stadt Wien 2018. „STEP 2025. Fachkonzept: Öffentlicher Raum“, hg. v. Magistratsabteilung 18 - Stadtentwicklung und Stadtplanung. https://tinyurl.com/d6ppydrj (28.8.2020).

Steer 2020. "Economic Impacts of Automous Delivery Services in the US." Final Report. New York. In https://www.steergroup.com/sites/default/files/2020-09/200910_\%20Nuro_Final_Report_Public.pdf (18.9.2020).

Umundum, P. 2020. „Die letzte Meile - Königsdisziplin der Logistik“, in Logistik - die unterschätzte Zukunftsindustrie. Strategien und Lösungen entlang der Supply Chain 4.0, hg. v. P. H. Voß, 149-162.

UPS 2020. „UPS And Waymo Partner To Begin Self-Driving Package Pickup In Arizona“, UPS Pressroom, 29.1.2020. https://pressroom.ups.com/pressroom/ContentDetailsViewer.page?ConceptType=PressReleases\&id=1580327674120-833 (19.2.2020).

USDOT (US Department of Transportation) 2020. „Nuro, Inc.; Receipt of Petition for Temporary Exemption for an Electric Vehicle with an Automated Driving System“. Washington D.C. www.nhtsa.gov/ sites/nhtsa.dot.gov/files/documents/nuro_notice_of_reciept_unofficial.pdf (19.2.2020).

Vogler, T., J.-P. Labus und O. Specht 2018. „Mögliche Auswirkungen von Digitalisierung auf die Organisation von Handelsunternehmen", in Digitalisierung im Handel. Geschäftsmodelle, Trends und Best Practice, hg. v. M. Knoppe und M. Wild. Wiesbaden: Gabler, 149-172.

Voß, P. H. 2020. Logistik - die unterschätzte Zukunftsindustrie. Strategien und Lösungen entlang der Supply Chain 4.0. Wiesbaden: Gabler.

WIFO (Österreichisches Institut für Wirtschaftsforschung) 2019. „Wie wird das Weihnachtsgeschäft 2019“, Pressekonferenz. Wien. https://tinyurl.com/e5frybts (16.3.2020)

Wiles, R. 2019. „Kroger ends its unmanned-vehicle grocery delivery pilot program in Arizona“, USA Today, 14.12.2019. https://tinyurl.com/m4syhubk (27.8.2020).

Wirtschaftsagentur Wien 2016. „City Logistik. Technologie Report“. Wien. https://wirtschaftsagentur. at/fileadmin/user_upload/Technologie/Factsheets_T-Reports/DE_CityLogistik_Technologie_Report.pdf (4.9.2020).

Wittenbrink, P., B. Leerkamp und T. Holthaus 2016. „Städtisches Güterverkehrskonzept Basel. Schlussbericht“. Basel: Bau- und Verkehrsdepartement des Kantons Basel-Stadt. Download unter www. mobilitaet.bs.ch/gesamtverkehr/verkehrskonzepte/gueterverkehrskonzept.html (26.8.2020).

Wittenhorst, T. 2019. „Deutsche Post stoppt Paketkästen für Privathäuser und Zustellroboter Postbot“, heise online, 3.2.2019. www.heise.de/newsticker/meldung/Deutsche-Post-stoppt-Paketkaestenfuer-Privathaeuser-und-Zustellroboter-Postbot-4296767.html (9.1.2020).

Wong, J. C. 2017. „Delivery robots: a revolutionary step or sidewalk-clogging nightmare?“, The Guardian, 12.4.2017. www.theguardian.com/technology/2017/apr/12/delivery-robots-doordash-yelp-sidewalk-problems (28.8.2020).

Open Access Dieses Beitrag wird unter der Creative Commons Namensnennung 4.0 International Lizenz (http://creativecommons.org/licenses/by/4.0/deed.de) veröffentlicht, welche die Nutzung, Vervielfältigung, Bearbeitung, Verbreitung und Wiedergabe in jeglichem Medium und Format erlaubt, sofern Sie den/die ursprünglichen Autor(en) und die Quelle ordnungsgemäß nennen, einen Link zur Creative Commons Lizenz beifügen und angeben, ob Änderungen vorgenommen wurden.

Die in diesem Beitrag enthaltenen Bilder und sonstiges Drittmaterial unterliegen ebenfalls der genannten Creative Commons Lizenz, sofern sich aus der Abbildungslegende nichts anderes ergibt. Sofern das betreffende Material nicht unter der genannten Creative Commons Lizenz steht und die betreffende Handlung nicht nach gesetzlichen Vorschriften erlaubt ist, ist für die oben aufgeführten Weiterverwendungen des Materials die Einwilligung des jeweiligen Rechteinhabers einzuholen.

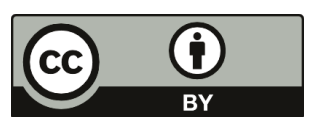

